\title{
CHEMISTRY OF DIABETES AND ITS IMPACT
}

\author{
Birendra Kumar Mishra \\ Department of Chemistry , TU, TRM Campus,Birgunj,Nepal \\ E-mail :- bkmishranepal@yahoo.co.in
}

\begin{abstract}
Diabetes mellitus is caused by defects in insulin production as will as action. In absence of insulin, the body's primary source of energy and the brains only source of energy, glucose is unable to enter into cells.Infact diabetes mellitus is a metabolic disease i.e. it is caused by an abnormal metabolism resulting from, for example nutritional deficiency, enzyme deficiency or abnormal secretion of hormones. In a case study among Nepalese people it has been found that diabetes mellitus is mainly caused by deterioration of renal function (Chronic Kidney Disease). Obesity and type 2 diabetes relation is an active and exciting area of research now a days. In this article an attempt has been made to overview the life threatening disease diabetes mellitus.
\end{abstract}

\section{Keywords}

Diabetes mellitus Insulin, Glucose ,Islets of Langerhens, AGEs,Glycated Hemogobin.

\section{Introduction}

In 2003, WHO Assistant Director- General, (Non communicable diseases and mental Health)said “ Diabetes is part of the growing epidemic of non communicable disease (NCDS) that are beginning to impose a double burden of disease on the world's poorest countries" Though these countries are struggling to address the problems of HIV/AIDS, malaria and tuberculosis, but now they must also prepare to deal with the onslaught of diseases that come with changes in life style and aging of their population. It is often said that these days generation are supposed to be less active than our parents and grandparents, people eat food with higher concentrations of sugar and fats often with the result of increasing weight of body and increasing the risk of diabetes. In China one in every 60 people is suffering from diabetes. A recent study shows that even among Chinese pre-school children, obesity rates increased 10-fold to affect one in every ten children over an 8-year period. This extra weight increases their risk of diabetes as they grow older. Nearly $6 \%$ of the U.S. population shows some 
degree of abnormality in glucose metabolism that is indicative of diabetes or tendency towards diabetes. It has been estimated that the number of cases of diabetes in developing countries is likely to increase more than two fold in the next thirty years from 115 million $2000 A$.D. to 280 million in 2030 A.D. According to Indian Diabetes Federation (IDF) and Madras Diabetes Foundation, India had 62.4 million with type 2 diabetes in 2011 in comparison to 50.8 million in 2010. About 9\% peoples are suffering from this disease and it may rise to $20 \%$ by 2030 in India. The IDF has predicted that India would have 100 million people with diabetes. (Shetty,2012) WHO report shows that diabetes has affected more than 436 thousand people in Nepal and this number will rise to 1,328 thousand by 2030 . In Nepal the percentage of diabetes patient has increased from $19.04 \%$ in 2002 to $25.9 \%$ in 2009 ( Dulal \& Karki, 2009 ). WHO has marked 14 Nov. as the Diabetes Day from 2003, with a commitment to increase its efforts to reduce the impact of diabetes and its complications, particularly among low and middle- income communities, in collaboration with the International Diabetes Federation (IDF).

Of course, diabetes is a common word which is used frequently to indicate the chronic common disease "diabetes mellitus" which means excessive excretion of sweet urine i.e. a condition in which excessive excretion of large amounts of glucose in the urine, a condition known as glucosuria. There are two major clinical types of diabetes mellitus: type1 diabetes also known as insulin dependent diabetes mellitus (IDDM) and type 2 diabetes mellitus or non-insulin dependent diabetes mellitus (NIDDM) known as insulin-resistant diabetes mellitus too. Diabetes mellitus is a metabolic disease i.e. it is caused by an abnormal metabolism resulting from, for example, nutritional deficiency, enzyme deficiency or abnormal secretion of hormones (Mayes, 1990). In a case study among Nepalese people it has been found that diabetes mellitus is mainly caused by deterioration of renal function (Chronic kidney disease) ( Mittal, et al.,2010)

\section{Causes of Diabetes}

Diabetes mellitus is caused by defects in insulin production as well as action. Type 1 diabetes begins early in life, and symptoms quickly become severe. This disease responds to insulin injection, because the metabolic defects stems from an autoimmunie destruction of pancreatic B-cells and a consequent inability to produce sufficient insulin which results in low rates of glucose uptake into muscle and adipose tissue. Type 1 diabetes requires both insulin therapy and careful, lifelong control of the balance between dietary intake and insulin dose. Characteristics symptoms of type 1 and type 2 diabetes are excessive thrust and frequent urination (polyuria) leading to the intake of large volume of water (polydipria). Type 2 diabetes is slow to develop often found in older, obese individuals and the symptoms are milder and often go unrecognized at first. This is really a group of diseases in which the regulatory activity of insulin is disordered: Insulin is produced but some feature of insulin response system is defective. Individuals with this order are insulin resistant. Without insulin the body's primary source of energy and the brains only source of energy, glucose is unable to enter at the cellular level. Few drugs impair insulin secretion or insulin action at its receptor site and potentially cause drugs induced diabetes. Though impairment in insulin secretion may not be sufficient to cause a patient to develop diabetes (Zavod, et al.,2008). The connection between type 2 diabetes and obesity is an active and exciting area of research now a days.

Individuals with either type of diabetes are unable to take up glucose efficiently from the blood. The characteristic metabolic change in diabetes is excessive but incomplete oxidation of fatty acids in the liver. The acetyl CoA produced by $\beta$-oxidation cannot be completely oxidized by the citric acid cycle because high 
$\left[\mathrm{NADH} / \mathrm{NAD}^{+}\right]$ratio produced by $\beta$-oxidation inhibits the cycle. Accumulation of high acetyl CoA leads to overproduction of the ketone bodies acetoacetate and $\beta$ - hydroxybutyrate which cannot be used by extrahepatic tissues as fast as they are made in the liver. In addition to $\beta$-hydroxy butyrate and acetoacetate, the blood of individuals with diabetes also contains acetone, which results from the spontaneous decarboxylation of acetoacetate. take place. Therefore one goal of providing therapy is to maintain the blood glucose level normal. The factors to maintain blood glucose are: balance of exercise, diet, insulin for the individual . Blood glucose concentration should be measured several times a day. Approximate adjustment of the amount of injected is also a major factor.

The concentration of glucose in blood and urine can be determined by fehling's reaction. Formerly this reaction was the basis for the diagnosis of diabetes. But in recent years a drop of blood is added to attest strip containing the enzyme glucose glucose oxidase.

The over production of ketone bodies, called ketosis results in greatly increased concentration of ketone bodies in the blood (ketonemia) and urine (ketonuria)

In uncontrolled diabetes the acid production by ketone overwhelm the capacity of the blood's bicarbonate buffering system and produce a lowering of blood $\mathrm{pH}$ called acidosis or ketoacidosis, which is a life threatening condition.

\section{Measurement of blood glucose in the diagnosis and treatment of Diabetes}

Glucose is the main fuel for the brain. When the amount of glucose reaching the brain is too low, the consequence can be dire: lethargy, coma, permanent brain damage and death. Blood glucose levels of $40 \mathrm{mg} / 100 \mathrm{ml}$ and below constitute severe hypoglycemia. For the satisfaction of brains need there must be enough concentration of glucose in the blood.

Person having IDDM, do not produce sufficient insulin for serving to reduce blood glucose concentration, with untreated diabetes may rise the blood glucose level to several fold higher than normal. Due to this kidney failure, cardiovascular disease, blindness and impaired wound healing can
After that a spectrophotometer measures the colour produced when $\mathrm{H}_{2} \mathrm{O}_{2}$ from glucose oxidation reacts with a dye indicating the blood glucose concentration.

$\mathrm{D}$-Glucose $+\mathrm{O}_{2} \longrightarrow$ D-Glucono- $\delta$-lactone $+\mathrm{H}_{2} \mathrm{O}_{2}$
$\mathrm{H}_{2} \mathrm{O}_{2}+$ Colorless
comp.

On the other hand blood glucose levels change with the timing of meals and exercise. Therefore single- time measurement do not predict the average blood glucose over hours and days, so dangerous increases may go undetected. The average glucose concentration can be measured by knowing its effect on hemoglobin because hemoglobin is always exposed to glucose at whatever concentration in the blood. A chemical reaction takes place in absence of any enzyme between glucose and amino group of hemoglobin. Thus, the amount of glycated hemoglobin present at any time gives the average blood glucose concentration. The extent of glycated hemoglobin is measured clinically by extracting hemoglobin from a small sample of blood using the technique of electrophoresis. Normal GHB values are about $5 \%$ of total hemoglobin which corresponds to the $120 \mathrm{mg} / 100 \mathrm{ml}$. The percentage of GHB increases to $13 \%$ in the persion with untreated 
diabetes. The value of GHB is maintained to $7 \%$ by insulin therapy in which the timing, frequency and dose of insulin injected are properly managed. During the hemoglobin glycation reaction Schiff's base is formed in the first step followed by rearrangement, oxidation and dehydration of the sugar moiety resulting a heterogeneous mixture of advanced glycation end products (AGES) e.g. $\varepsilon \mathrm{N}$-carboxy methyl lysine and methylglyoxal etc. These end products can leave the erothrocyte and crosslinks covalently between proteins and interferes in the normal function of proteins which causes the damage of kidneys, retinas and cardiovascular system (Scheme 1).
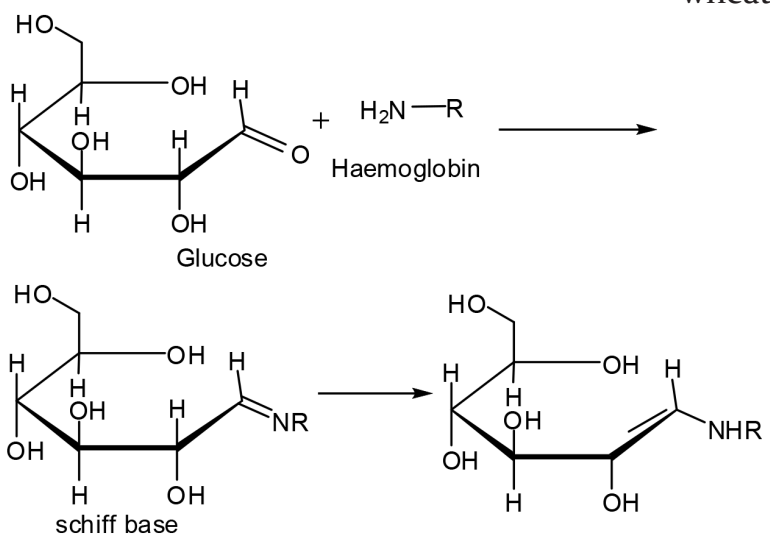

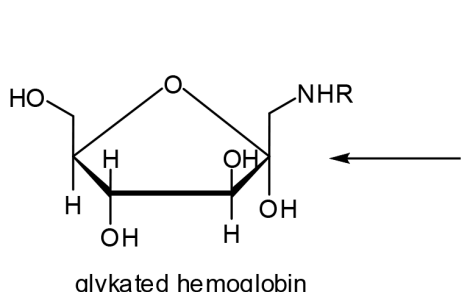

(GHB)

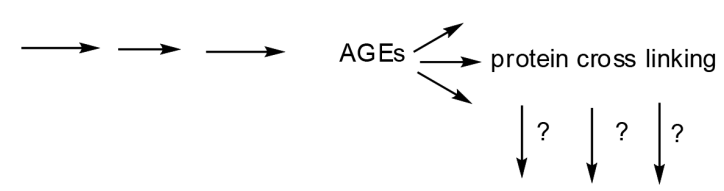

Damage to kidney, retina \&cardiovascular system

scheme - 1 : Non enzymatic reaction of glucose with hemoglobin

\section{Insulin counters high blood glucose}

The adjustment of blood glucose level near $4.5 \mathrm{~mm}$ involve the combined action of insulin, glucagon, epinephrine and cortisol on metabolic processes in many body tissues, especially in lever muscle and adipose tissue. In fact, the effect of insulin is to favor the conversion of excess blood glucose to two storage forms: (i) in the liver and muscle as glycogen (ii) in adipose tissue as triacyl glycerols (TGA). Besides this direct effect, insulin can also act indirectly in brain to signal these tissues.

Pancreatic $\beta$ cells secrete insulin in response to changes in blood glucose. When a person takes a carbohydrate- rich meal (like rice, wheat, sweets etc.) glucose enters the blood stream via intestine, then blood glucose increases, so that secretion of insulin is increased by the pancreas. It is largely regulated by the level of glucose in the blood supplying the pancreas. The peptide hormones like insulin, glucagon and somato statin are produced by clusters of specialized pancreatic cells, the islets of langerhans. Each cell type of the islets produce a specific peptide homone e.g. $\alpha$-cells produce glucagon, $\beta$-cells insulin and $\delta$-cells, somatostatin.

When the amount of glucose rises in blood, active metabolism of glucose in the $\beta$-cell increases intracellular concentration of Adinocine Triphosphate (ATP) which closes $\mathrm{k}^{+}$channels in the plasma membrane. Due to the change in membrane potential, voltage-gated $\mathrm{Ca}^{++}$channels open which allows the $\mathrm{Ca}^{++}$to flow into the cell. At the same time $\mathrm{Ca}^{++}$is also released from the endoplasmic reticulum in response to the initial increasement in the concentration of $\mathrm{Ca}^{++}$to the cytosol. This concentration is enough to trigger insulin release by exocytosis. (Fig.1) 


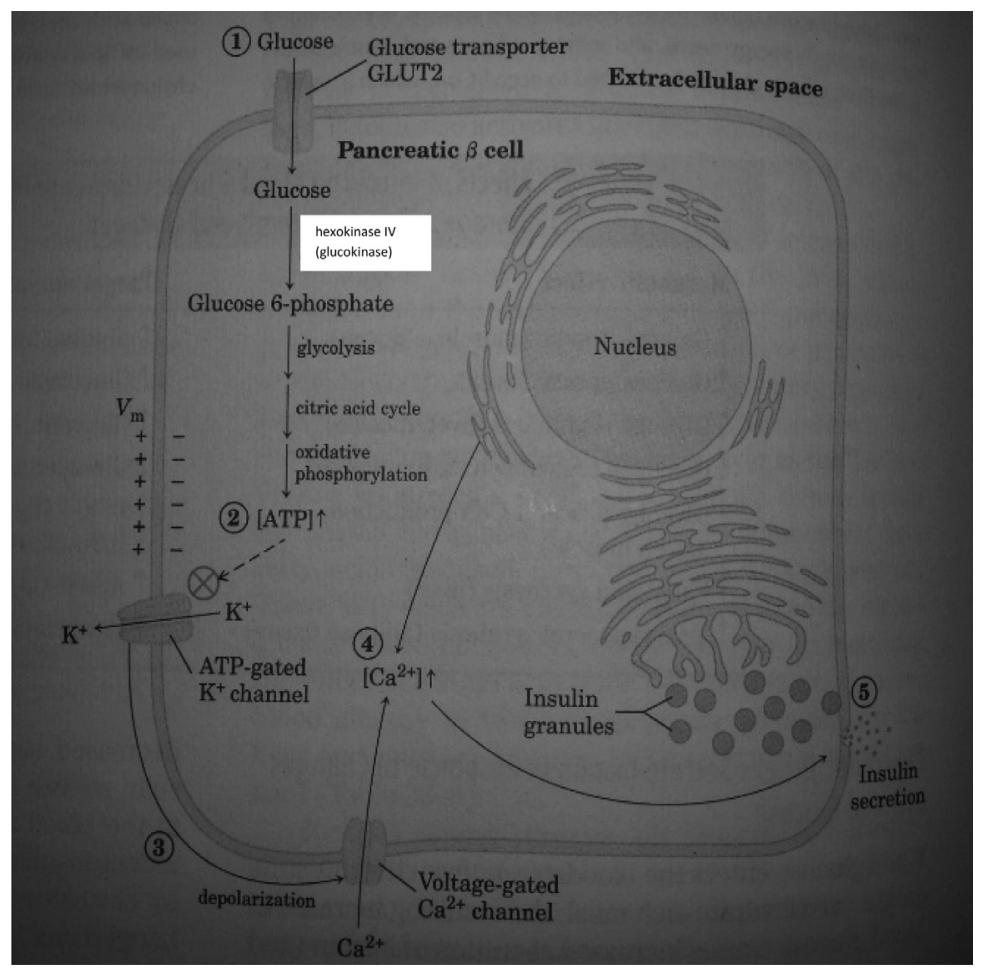

Fig: 1 Glucose regulation of insulin secretion in a pancreatic $\beta$ cell

Source: Principles of Biochemistry (5ed) W.H Freeman and Company, Newyork become the principle fuel .The liver converts the fatty acid to ketone bodies export to other tissues including the brain.

\section{Obesity and type 2 diabetes}

Obesity and associated type type 2 diabetes have increased globally in individuals of diverse ethnic and cultural backgrounds. Technical advances in molecular biology have revailed intense search for genetic defects responsible for the development and maintenance for obesity. This search has led to the identification of a few genes that are associated with or responsible for obesity. In spite of, advancement about the genetics of obesity, this information has not yet been translated into effective and

There are many drugs which stimulate insulin secretion, but the sulphonylurea compounds are used most frequently for therapy in human. Drugs such as tolbutamide stimulate insulin release by a mechanism different from that employed by glucose and have achieved by wide spread use in the treatment of type 2 diabetes.

Several hours after the meal, blood glucose level fall slightly due to the ongoing oxidation of glucose by the brain and other tissues. This lowered blood glucose triggers secretion of glucagon and decreases insulin release. During the long gap of dietary intake the liver becomes the main source of glucose for brain liver glucose is broken down and the glucose -1- Phosphate produced is converted to glucose -6- phosphate and then to free glucose which is released in the blood stream. In prolonged fasting or starvation triacylglycerols (TAGs) practical means of the treatment of such disorders. On the other hand, observations of vertebrates in the wild indicate that obesity has evolved as a strategy to survive long periods of low food availability. During such periods fat provides a lasting energy source and insulin resistance supports glucose supply to the brain. In this respect, the seasonal obesity is regulated by endogenous factors controlling body composition. Evidence now exists that this annual cycle of obesity is the net result of changing interactions of multiple circadian neural occcilation within the central Obesity and overweight is defined in terms of body mass index (BMI) which is taken as the ratio of weight in $\mathrm{kg}$ and square the height in ' $m$ '. A BMI below 25 is said to be normal. An individual with a BMI of 25 to 30 is overweight and BMI greater than 30 indicates obesity. nervous system. (Meier \& Cincotta, 1996). 
Obesity provides the chances of developing type 2 diabetes as well as heart attack, stroke and cancers of the colon, breast, prostate and endometrium . Therefore to understand the regulation of body mass and the storage of fat in adipose tissue is very important. An approximation is made that obesity is the concurring activities. The excess calories are managed in three ways by the body.

(1) Conversion of excess fuel to fat and string in adipose tissue (2) burning of excess fuel by extra exercise and (3) wasting the fuel by diverting to heat production by uncoupled mitochondria at the cellular level. A complex set of hormonal and neuronal signals balance fuel in take and energy expenditure, so as to hold the amount of adipose tissue at a requires understanding these various checks and balances under normal condition. Since the main characteristic of type 2 diabetes is the development of insulin resistance, therefore more insulin is needed to bring about the biological effects produced by a lower amount of insulin in the normal healthy state. In the beginning of type.2 diabetes pancreatic B cells secrete enough insulin to overcome the lower insulin sensitivity of muscle and liver. But lastly $\mathrm{B}$ - cells fail and the lack of insulin apparent in the body's ability to regulate blood glucose the intermediate stage before type 2 diabetes is called the metabolic syndrome.This is typified by obesity specially in the abdomen high blood pressure, abnormal blood lipids i.e. high TAG \& LDL with low HDL; slightly high blood glucose and a reduced ability to clear glucose in the glucose - tolerance test (Cox \& Nelson, 2010). Person with metabolic syndrome often show changes in blood proteins which is associated with abnormal clothing .Genetic factors are also responsible to predispose toward type 2 diabetes. Although $80 \%$ of type 2 diabetes are obese. Some obese individuals do not develop type 2 diabetes also.

\section{Conclusion}

It has been estimated that $150-170$ million people worldwide are suffering from diabetes mainly of type 2 . This number may reach 220 million sin 2010 and 360 million in 2030. In the industrialized world there is growing epidemic of obesity and the type 2 diabetes due to more than adequate food supply. The pathology of diabetes includes cardiovascular disease, renal failure, blindness etc. The global mortality from diabetes was 2.9 million in 2000 A.D. and it is sure to rise this number in coming years. Therefore relationship between type 2 diabetes and obesity study is essential to prevent the damage due to this disease. The disease ,type 2 diabetes can be managed properly with dietary restriction with accompanying weight loss,regular exercise and drugs (medication) that increase insulin sensitivity or insulin production .

In a research it has been shown that bromocriptine argot alkaloid following moderate hypocaloric diet improves glucose - tolerance and promotes significant weight and body fat loss in obsess subsets over an 18 week treatment period ( Cincotta \& Meier, 1996).

\section{Reference}

Cincotta A.H. \& meier A.H and Cincotta , A.H. (1996).Bromocriptine (Ergo set) Reduces body weight \& improves glucose toluene in obese subjects, Diabetes Care , 19 (6) , $667-670$.

Cox, M.M. \& NelsonD.L. (2010). Principles of Biochemistry, Newyork, W.H Freeman \& company.

Dulal, R. K. \& Karki, S. (2009). Disease management programme for diabetes mellitus in Nepal. J. of Nep. Mdical Assoc. 48 (176) $281-286$

Mayes P.A. (1990) in Harper' Biochemistry (22 ed.) prentice-Hall international Inc.

Meier,A.H.\& and Cinotta, A.H. (1996). Circadian rhythms regulate the expression of the thrifty genotype /phenotype .Diabetes Reviews, 4(4), 464 - 486. 
Mittal, A., Sathian, B., Kumar,A., Chandrasekharan, N. \& Suka, A (2010). Diabetes mellitus as a potential risk factor for renal disease among Nepalese . A hospital based case control study . Nepal Journal of epidemiology, 1 (1),22,-22.

Shetty,P.(2012). Epigenetics and life style are conspiring to inflict a massive epedimic of type 2 diabetes in the subcontinemt. Nature 485 514-516 (17 May 2012).
Zavod, R.M.,Krstenansky J.L. \& Currie B.L (2008). Insulin \& drugs used for the treatment of diabetes. In Foye's Medicinal Chemistry ( $6^{\text {th }}$ ed.) Baltimore, Lippincott Williams Wilkins. 